FOCAL hand dystonia involves a loss of motor control of one or more digits; it is associated with the repetitive, synchronous movements of the digits made by musicians over periods of many years. Magnetic source imaging revealed that there is a smaller distance (fusion) between the representations of the digits in somatosensory cortex for the affected hand of dystonic musicians than for the hands of non-musician control subjects. The data suggest that use-dependent susceptibility to digital representation fusion in cortex may be involved in the etiology of focal dystonia. A successful therapy for the condition has been developed based on this consideration. NeuroRepor t9: 3571-3575 (C) 1998 Lippincott Williams \& Wilkins.

Key word: Cortex; Focal dystonia; Magnetic source imaging; Magnetoencephalography (MEG); Plasticity; Reorganization; Somatotopy

\section{Alteration of digital representations in somatosensory cortex in focal hand dystonia}

\author{
Thomas Elbert, ${ }^{\mathrm{CA}}$ Victor Candia, \\ Eckart Altenmüller, ${ }^{1}$ Harald Rau, \\ Annette Sterr, Brigitte Rockstroh, \\ Christo Pantev ${ }^{2}$ and Edward Taub ${ }^{3}$
}

\author{
Department of Psychology, University of \\ Konstanz, D-78457, Konstanz; ${ }^{1}$ Institut für \\ Musikphysiologie und Musiker-Medizin \\ Hochschule für Musik und Theater, Plathnerstr. \\ 35, D-30175 Hannover; ${ }^{2}$ Institute of \\ Experimental Audiology, University of Münster, \\ Kardinal-von-Galen-Ring 10, D-48129 Münster, \\ Germany; ${ }^{3}$ Department of Psychology $415 \mathrm{CH}$, \\ University of Alabama at Birmingham, \\ AL 35294, USA
}

${ }^{\mathrm{CA} C}$ Corresponding Author

\section{Introduction}

Focal hand dystonia of musicians is a painless motor disorder involving loss of control of individual finger movements. It often manifests in the third to the fifth digits (mid-to-little finger, D3-D5), but it can be present in any of the digits; two or more adjacent digits are usually involved. The condition can occur in pianists, organists, wind players, guitarists, and other string players and is thought to be associated with the many hours of daily practice engaged in by professional musicians beginning at the often early age at which they initiate their musical education. ${ }^{1-3}$

The disorder has been reported to affect $14-16 \%$ of musicians ${ }^{2}$ who seek treatment for hand problems. ${ }^{2,4}$ However, diagnosis is sometimes problematic, ${ }^{2}$ and therefore the actual prevalence is difficult to estimate unambiguously. It appears to be related to other conditions associated with prolonged performance of rapid, alternating and/or forceful movements of the digits occurring in such occupational activities as typing, data processing and device assembly. ${ }^{5}$ The disorder is usually idiopathic; no clear cause in terms of the amount or biomechanics of practice and no physiological mechanism has yet been determined. ${ }^{1,6,7}$ Similarly, no treatment has yet been found that has anything but a temporary palliative effect. ${ }^{1-3}$

In seminal work, By $\notin t$ al. developed a presumed animal model of focal hand dystonia in owl monkeys involving the frequent repetition of grasp over an extended period. ${ }^{8,9}$ Under these circumstances, the monkeys developed motor disturbances and many distortions of the receptive fields and representational zones of the digits in area $3 \mathrm{~b}$ of the somatosensory cortex, particularly a breakdown in and reordering of their boundaries as well as the emergence of new cortical zones representing portions of the hand not normally included in the same zone. Wang and coworkers ${ }^{10}$ had shown in work from the same laboratory that extensive simultaneous stimulation of just the distal or proximal phalanges of D2-4 in new world monkeys led to the development of anomalous new multi-digit receptive fields responsive to just the intensively stimulated parts of the hand. Moreover, surgically joining two digits in monkeys resulted in a fused cortical receptive field for those two digits, ${ }^{11,12}$ while surgical separation of digits in syndactylous 
humans led to a separation of the cortical representational zones of the digits. ${ }^{13}$ Similarly, Sterr et al. ${ }^{14,15}$ found that the cortical representation of the digits became topographically disordered in blind individuals who read Braille with three fingers simultaneously for many hours on a daily basis.

Thus, extensive simultaneous stimulation of the digits and other types of prolonged, unusual types of sensory input can produce a use-dependent reorganization of digital receptive fields. The purpose of the present study was to use magnetic source imaging to test the possibility that musicians with focal hand dystonia have greater fusion (i.e. shorter interdigital distances) between the representational zones of the fingers of the dystonic hand than is the case for the digital cortical representations of non-dystonic musicians or non-musician control subjects.

\section{Materials and Methods}

Eight right-handed professional musicians (5 males) suffering from focal hand dystonia, eight unaffected musicians and nine non-musician controls gave written consent to participation in the study. Each dystonic patient was referred from the clinical practice of E.A. and given a thorough neurological examination before project intake. An exclusion criterion was that there be no neurological condition other than the focal hand dystonia. The non-dystonic musician controls were matched to the dystonic subjects on instruments played and age. Some demographic and clinical characteristics of the dystonic subjects are presented in Table 1 . The study procedure was approved by the university's ethics review board.

Somatosensory stimulation consisted of light superficial pressure applied by a pneumatic stimulator using a standard non-painful stimulation intensity. ${ }^{16}$ Tactile stimulation was delivered to the midvolar aspect of the distal phalanx of all digits of both hands in dystonic and non-dystonic musicians and to left and right D1, D2, and D5 in normal control subjects. At each site, 1000 stimuli were given at an average rate of $0.5 \mathrm{~Hz}$. The interval

Table 1. Characteristics of musicians with focal dystonia

\begin{tabular}{lllcc}
\hline Subject & $\begin{array}{l}\text { Instru- } \\
\text { ment }\end{array}$ & $\begin{array}{l}\text { Affected } \\
\text { digits }\end{array}$ & $\begin{array}{c}\text { Age } \\
\text { (years) }\end{array}$ & $\begin{array}{c}\text { Chronicity } \\
\text { (years) }\end{array}$ \\
\hline G1 & Guitar & left D3,D4,D5 & 30 & 5 \\
G3 & Guitar & right D3,D4,D5 & 36 & 3 \\
OB & Oboe & right D3,D4,D5 & 38 & 3 \\
OR & Organ & left D3,D4,D5 & 47 & 3 \\
CL & Clarinet & right D3,D4 & 28 & 1 \\
FL & Flute & left D2,D3,D4 & 42 & 4 \\
KL & Piano & right D2,D3,D4,D5 & 25 & 4 \\
G2 & & & & \\
(excluded) & Guitar & right D1,D2 & 42 & 13 \\
\hline
\end{tabular}

between stimulus onsets varied randomly between 450 and $550 \mathrm{~ms}$. Each hand was tested separately with order of hand tested counterbalanced across subjects. Stimulation site within each hand was varied according to a pseudorandom order.

The sensor array of magnetic detectors (BTi Magnes 37-channel biomagnetometer) was positioned over the hemisphere contralateral to the stimulated fingers centered over C3 or C4. Data were filtered with a bandpass of $0.1-200 \mathrm{~Hz}$ and sampled at a rate of $520.5 \mathrm{~Hz}$. A response was omitted from the average if its range exceeded $2 \mathrm{pT}$ in any of the MEG channels.

For the source analysis, responses were filtered from 1 to $20 \mathrm{~Hz}$. A first major peak was identified in each of the evoked waveforms within the time window of $30-75 \mathrm{~ms}^{16-18}$ For this peak, a single equivalent current dipole (ECD) model (using the best fitting local sphere) was fitted to the measured field distribution and the medians of the dipole moment and the dipole location were computed from a selection of contiguous time points within a $20 \mathrm{~ms}$ time segment (11 sampling points) around the maximal root mean square (RMS) across the 37 channels. Points were selected if they met the following criteria: (1) RMS indicating a signal-to-noise ratio > 3 , (2) goodness of fit of the ECD-model to the measured field $>0.95$, and (3) a minimal confidence volume of the ECD location $<300 \mathrm{~mm}^{3}$. (In dystonic subject G2, the record for one of the dystonic fingers did not conform to these criteria and this individual's data were therefore excluded from further analysis.) It has been shown that the first major peak of the somatosensory evoked magnetic field has its source in the primary somatosensory representation (SI), with most of the activity originating in Brodman's area $3 \mathrm{~b}$ contralateral to the stimulation side. ${ }^{19}$

As a measure of the medial-lateral extent of the territory covered by the cortical digit representations, the following digit representation fusion index (DRFI) was computed:

$$
\text { DRFI }=\sqrt{(D 5-D 2)^{2}+(D 2-D 1)^{2}+(D 5-D 2)^{2}}
$$

Squaring the interdigital distances has the effect of increasing the influence of large distances between the cortical representation of the fingers. Thus, the smaller the DRFI, the greater the digital fusion. The DRFI values were transformed to $\mathrm{z}$-scores using the values observed in the normal control subjects as the reference. This was done by taking the difference between the DFRI score for each musician and the average of the DRFI scores for all nine control subjects and dividing by the standard deviation of the scores for controls. These $\mathrm{z}$-scores were submitted to an ANOVA comprising the within subjects factor HAND and the factor GROUP. 
The data were also analysed using a previously published measure of the topographic order/disorder of the digital representations in primary somatosensory cortex. ${ }^{15}$ For this measure, all topographic arrangements in which the cortical representation of the digits are in the correct order in the inferiorsuperior dimension (D1 $\rightarrow \mathrm{D} 2 \rightarrow \mathrm{D} 5)$ are assigned a value of zero. If there is topographic disorder in any pair of cortical digital representations (D1-D2, D2-D5, or D1-D5), each of the distances in the deviant direction are summed and placed in the nominator of a fraction whose denominator is the distance between representations of the two fingers most distant from one another. Using the size of the cortical representation of the hand as a denominator serves to correct for the fact that there may be an expansion of the hand representation in the musicians compared with the controls; ${ }^{17}$ without this correction the amount of cortical disorder would be overestimated for the two musician groups. The measure can be calculated as follows:

$$
\begin{aligned}
& \text { Cortical disorder }= \\
& \quad\{[\text { deviance }(D 1-D 2)]+[\text { deviance }(D 1-D 5)] \\
& \quad+[\text { deviance }(D 2-D 5)]\} / \text { distance between } \\
& \text { farthest apart finger representations }
\end{aligned}
$$

\section{Results}

The DRFI or fusion scores (Fig. 1) show that the distance between the cortical representation of the digits was significantly smaller, averaged across the two hands, in the dystonic musicians than in the two hands of the non-musician controls $(\mathrm{z}=-0.80, \mathrm{t}(6)=$ $3.7, p<0.01)$. Moreover, there was significantly more fusion in the digital representation of the digits of the dystonic hand than in both hands of the nonmusician controls $(\mathrm{z}=-8.7, \mathrm{t}(6)=-3.5, p<0.02)$; the same difference was not significant for the nondystonic hand $(\mathrm{z}=-0.73, \mathrm{t}(6)=-1.7, p=0.14)$. The DRFI score (both hands) for the non-dystonic musicians was nearly the same as that of the nonmusician controls $(z=0.08)$.

Figure 2A illustrates the fusion of the digital cortical representations opposite the affected hand in a dystonic musician; the spacing of the digital representations opposite the non-dystonic hand is normal. Figure 2B shows digital fusion in the hemisphere opposite the dystonic hand in another musician; in this case, however, there was an equal amount of fusion in the hemisphere opposite the non-dystonic hand. This phenomenon was observed in four of the seven dystonic musicians.

The cortical disorder score was not significantly greater for the dystonic musicians than for the nondystonic musicians (both hands), for the affected

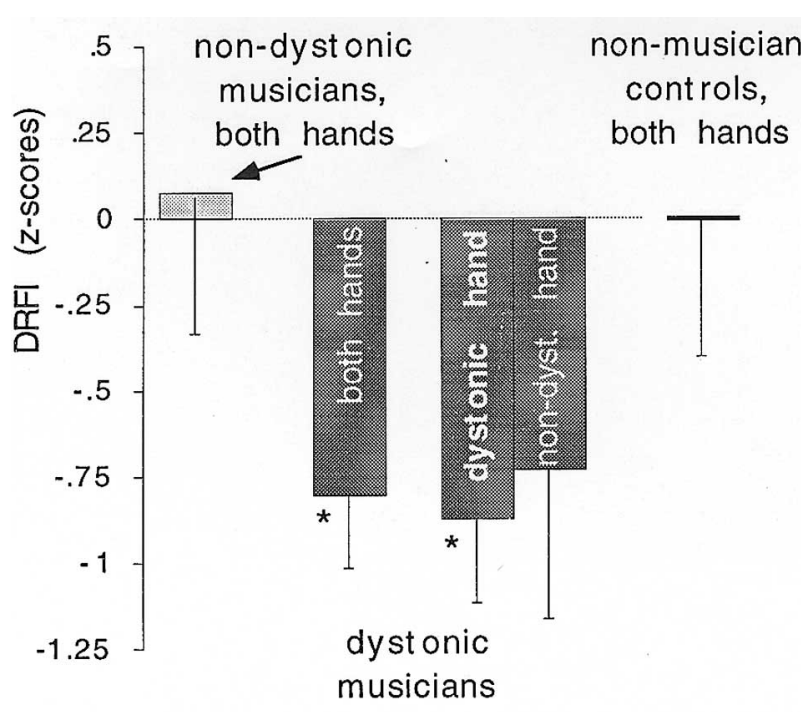

FIG. 1. Dystonia representation fusion index (DFRI) scores converted to z-scores (mean and standard errors) for the dystonic hand, the non-dystonic hand and the mean of both hands in musicians with focal dystonia and for both hands in non-dystonic musicians and non-musician control subjects. Values were transformed to z-scores using the DRFI-values observed in the non-musician control subjects as the reference (i.e. normal controls have a mean of zero, a standard deviation of 1 , and a standard error of 0.33 ). ${ }^{*} p<0.05$ compared with non-musician control subjects.

hand representation in dystonic musicians compared with both hand representations in the non-dystonic musicians, or for the affected hand representation compared with the non-dystonic hand representation in dystonic musicians. Thus, focal hand dystonia appears to be associated with a fusion of the cortical representations of the digits and not a disarrangement of their topographic order.

\section{Discussion}

There is a reduced distance between the representational zones of the digits in primary somatosensory cortex for the affected hand of dystonic musicians compared with the representations of the digits in non-musician control subjects. This constitutes a change that is in the direction of fusion of the digital receptive fields. Such a change also occurred in the cortex opposite the non-dystonic hand in four of seven of the dystonic musicians studied. The effect in this hemisphere was not statistically significant on a group basis because three subjects did not exhibit the phenomenon. However, the observation is consistent with the fact that some string players who change the hand used for fingering the strings after developing focal dystonia, also develop dystonia in the hand to which they switch (data from the clinical practice of E.A.). Changes in corticomotor representation, assessed by transcranial magnetic stimulation, have also been reported, ${ }^{20}$ consisting of distortions of the shape of the motor hand area, 


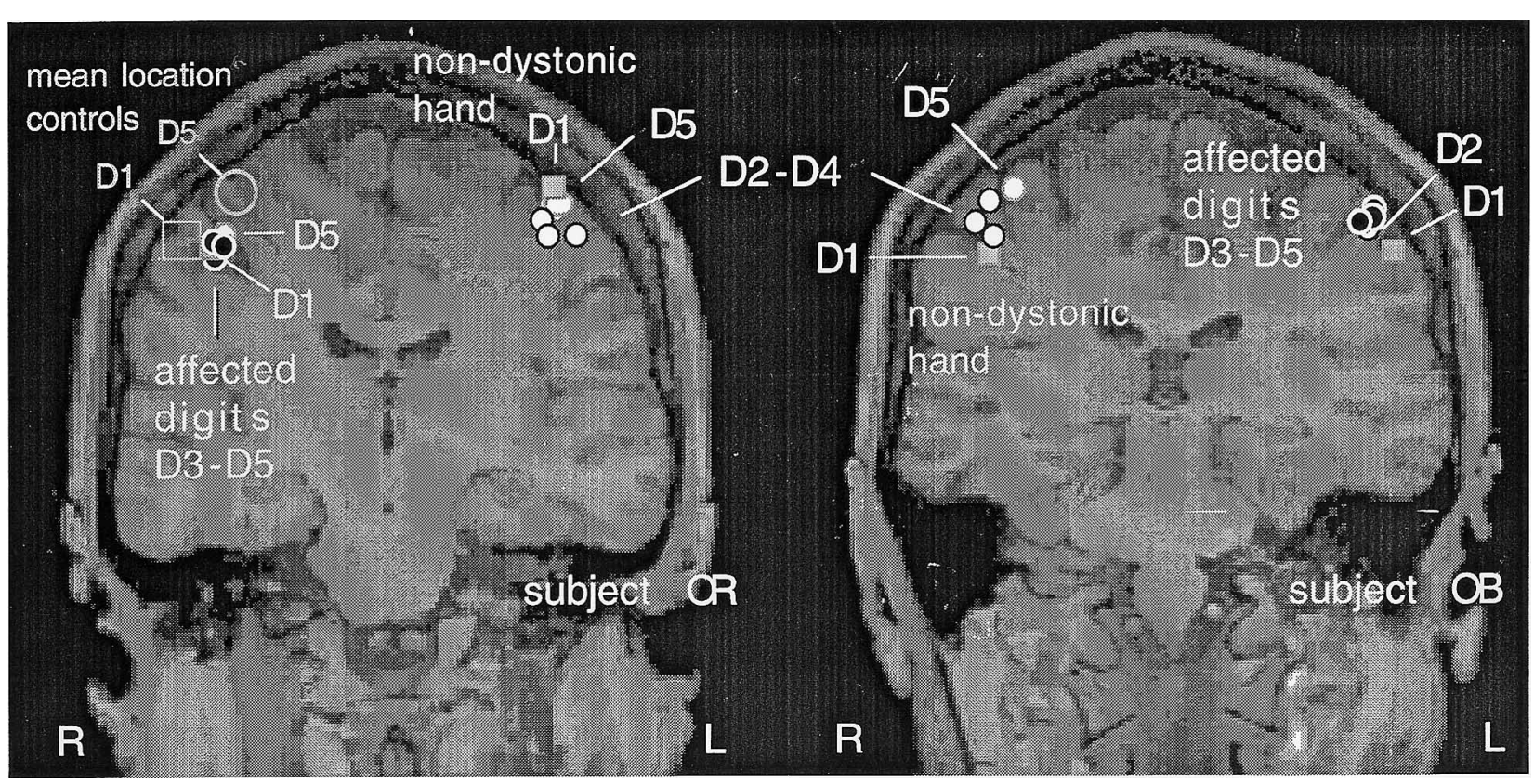

FIG. 2. A coronal MRI section through the somatosensory cortices of 2 musicians (OR and OB) with focal hand dystonia onto which are projected the dipole locations of digits 1-5 (D1-D5) resulting from contralateral stimulation. The large open symbols in the right hemisphere of subject OR indicate the mean location of dipoles for D1 and D5 in normal control subjects.

extension of its lateral borders and the emergence of almost discrete secondary motor areas. However, it is currently unclear to what extent these distortions of the motor map and the alterations in the sensory map observed here are related.

The cross-sectional data obtained in this study does not permit causal attribution. It is possible that the cortical fusion was involved with causation in the development of the dystonia, however, it is also possible that the reverse was true and that the dystonia, resulting from some other cause or set of causes, produced the cortical fusion. Another possibility is that some third factor(s), such as a functional disturbance of the basal ganglia ${ }^{21}$ caused both the dystonia and the tendency toward cortical digital fusion.

If a tendency toward cortical digital fusion is a factor in the genesis of focal hand dystonia, one might hypothesize that an intervention that served to break apart the fusion would constitute an effective therapy for the condition. This might be accomplished by a sensory learning process in which dystonia patients were given extensive experience in making complex tactile discriminations with individual fingers, as suggested by Byl et al. ${ }^{8}$ Alternatively, since focal hand dystonia is a disturbance of motor production, one might fashion a therapy in which patients were given extensive practice in making discrete individuated finger movements. A treatment based on this principle in combination with methods employed in constraint-induced (CI) movement therapy, an effective new intervention for rehabilitation of movement after stroke, ${ }^{22-24}$ has been developed in this laboratory. It has proved to be effective in the four pianists and two classical guitarists with focal hand dystonia treated to date (Candia V., Elbert T., Altenmüller, E., Rau, H., Schäfer, T. and Raub, E., unpublished data); Note: Another pianist finished a highly successful course of treatment after submission of the manuscript).

\section{Conclusion}

Most professional musicians engage in extensive practice of their instrument, especially during the formative period when they are students. Why does one musician develop focal dystonia when another does not who presumably carries out as much repetitive, forceful and highly articulated movement of several fingers synchronously? If the first possibility noted above were correct, and cortical digital fusion in response to repetitive finger movement is a causal factor in the etiology of the motor disturbance, it would be possible that individuals who are susceptible to the development of focal dystonia are those who have a prior tendency toward cortical digital fusion. The degree of susceptibility would be correlated with the degree to which an individual was liable to exhibit the digital fusion-type of use-dependent cortical plasticity.

\section{References}

1. Jankovic J and Shale H. Semin Neurol 9, 131-135 (1989).

2. Lederman RJ. Med Probl Perf Art 3, 45-51 (1988).

3. Beijani FJ, Kaye GM and Benham M. Med Probl Perf Art 77, 406-413 (1996).

4. Hochberg FH, Lavin P, Portnoy R et al. Arch Phys Med Rehabil 3, 9-14 (1988). 
5. Sheehy MP and Marsden CD. Brain 105, 461-480 (1982).

6. Cohen LG and Hallett M. Neurology 38, 1005-1012 (1988).

7. Marsden CD. Med Probl Perf Art 6, 116-121 (1991).

8. Byl NN, Merzenich MM and Jenkins WM. Neurology 47, 508-520 (1996).

9. Byl NN, Merzenich MM, Cheung S et al. Phys Ther 77, 269-284 (1997).

10. Wang X, Merzenich MM, Sameshima K et al. Nature 378, 71-75 (1995).

11. Clark SA, Allard T, Jenkins WM et al. Nature 332, 444-445 (1988).

12. Allard T, Clark SA, Jenkins WM et al. J Neurophysiol 66, 1048-1058 (1991).

13. Mogilner A, Grossmann JA, Ribary U et al. Proc Natl Acad Sci 90, 3593-3597 (1993)

14. Sterr A Müller MM, Elbert T et al Nature 391, 134-135 (1998).

15. Sterr A, Müller MM, Elbert T et al. J Neurosci 18, 4417-4423 (1998).

16. Elbert T, Flor H, Birbaumer N et al. NeuroReport 5, 2593-2597 (1994).

17. Elbert T, Pantev C, Wienbruch C et al. Science 220, 21-23 (1995).

18. Flor H, Elbert T, Knecht $S$ et al. Nature 375, 482-484 (1995).

19. Baumgartner C, Doppelbauer A, Deecke L et al. Exp Brain Res 87, 641-648 (1991).

20. Byrnes ML, Thickbroom GW, Wilson SA et al. Brain 121, 977-988 (1988).
21. Berardelli A, Tothwell JC, Hallet $M$ et al. Brain 121, 1195-1212 (1998).

22. Taub E. Somatosensory of deafferentation research with monkeys: Implications for rehabilitation medicine. In: Ince LP, ed. Behavioral Psychology in Rehabilitation Medicine: Clinical Applications. New York: Williams \& Wilkins, 1980: 371-401.

23. Taub E, Miller NE, Novack TA et al. Arch Phys Med Rehabil 74, 347-354 (1993)

24. Taub E, Crago JE and Uswatte G. Rehabil Psychol 43, 152-170 (1998).

ACKNOWLEDGEMENTS: This work was supported by grants from the Deutsche Forschungsgemeinschaft and Grant B95-975R from the Rehabilitation Research and Development Service, U.S. Department of Veterans Affairs.

\section{Received 19 August 1998; accepted 1 September 1998}

